\title{
CLÁSICA ARQUEOLOGÍA, ANTIGUA HISTORIA: ENSAYO EN TORNO A UN DESENCUENTRO EN LA TRADICIÓN HISTORIOGRÁFICA DE ANDALUCÎA OCCIDENTAL
}

\author{
CLASSICAL ARCHAEOLOGY AND ANCIENT HISTORY: ESSAY ON A FAILED \\ MEETING IN THE HISTORIOGRAPHIC TRADITION OF WESTERN ANDALUSIA
}

\author{
por \\ SILVIA FERNÁNDEZ CACHO \\ LEONARDO GARCÍA SANJUÁN
}

\begin{abstract}
RESUMEN: Desde una perspectiva crítica, este trabajo realiza un ensayo de valoración historiográfica y teórica del paradigma de investigación predominante en las áreas de Arqueología Clásica e Historia Antigua en Andalucía Occidental durante los últimos veinte años.
\end{abstract}

ABSTRACT: This paper assesses the traditional research paradigm predominating in both Classical Archaelogy and Ancient History in Western Andalusia over the last twenty years.

Este trabajo parte de la observación de que en la situación actual, el estudio de la Historia Antigua en Andalucía Occidental se encuentra prisionero de una contradicción con la que tropieza cualquier licenciado dispuesto tanto a iniciar estudios de postgraduado en la materia como a desarrollar una labor profesional en un medio no académico. Desde una perspectiva historiográfica crítica ${ }^{1}$, se examina a continuación la evolución reciente de las denominadas "Historia Antigua" y "Arqueología Clásica" en dicho medio académico.

1. En Arqueología se ha concebido frecuentemente historiografía como relación de actuaciones de investigación, lo cual la ha reducido a un mero listado acrítico de descubrimientos fundamentales, excavaciones importantes, publicaciones señeras o incluso aspectos biográficos y curriculares carente de una discusión de las teorias arqueológicas a cuya construcción aquellos estaban contribuyendo. Aquí se entiende historiografía en lo que G. Pasamar (1991) denomina «dimensión teórica». 


\section{LA SITUACIÓN ACTUAL}

Para evaluar las tendencias predominantes en la investigación de la Historia Antigua de Andalucía durante las últimas dos décadas se ha realizado un estudio cuantitativo sencillo de los trabajos publicados en ocho revistas especializadas, publicadas por otras tantas instituciones andaluzas vinculadas de una u otra forma a las ciencias sociales e históricas. Estas revistas son:

- Baetica. Estudios de Arte, Geografía e Historia. Facultad de Filosofía y Letras de la Universidad de Málaga. (1978-1991)

- Mainake. Area de Cultura de la Diputación Provincial de Málaga. (1979-1990)

- Habis. Facultad de Geografía e Historia de la Universidad de Sevilla. (1970-1991)

- Corduba. Revista del Museo Arqueológico Provincial de Córdoba. Diputación Provincial de Córdoba. (1976-1985)

- Ariadna. Revista de Investigación del Museo Municipal de Palma del Río (Córdoba). Area de Cultura del Ayuntamiento de Palma del Río. (1987-1991)

- Gades. Colegio Universitario de Filosofía y Letras de Cádiz. (1978-1988)

- Almoraima. Revista de Estudios Campogibraltareños. Mancomunidad de Municipios del Campo de Gibraltar. (1988-1991)

- Huelva Arqueológica. Diputación Provincial de Huelva. (1975-1987)

Dentro del conjunto de trabajos examinados (276 en total), que se encuentran por supuesto muy irregularmente distribuidos en dichas revistas, por una parte debido a que el grado de especialización en torno a la Historia Antigua de unas y otras difiere sensiblemente, y por otra a su variable antigüedad, se ha realizado una clasificación temática, de acuerdo con el siguiente esquema:

\section{DESCRIPCIONES}

- Monumentos

- Informes de Prospecciones/Excavaciones

- Mosaicos/Pinturas

- Cerámicas

- Esculturas

- Epígrafes

- Monedas

- Otros artefactos (terracotas, útiles óseos, etc..)

\section{INTERPRETACIONES DATOS EPIGRÁFICO-LITERARIOS}

- Paleogeografía/Paleoambiente/Recursos Naturales

- Demografía

- Estructura socioeconómica

- Organización Política

- Organización administrativo/territorial

- Estructura ideológico/religiosa

- Conflictos bélicos (batallas/invasiones/campañas)

- Ciudades (identificación/localización/comentario) 


\section{INTERPRETACIONES DATOS ARQUEOLÓGICOS}

- Paleogeografía/Paleoambiente/Recursos Naturales

- Demografía/Paleoantropología

- Estructura socioeconómica

- Organización política

- Organización territorial (análisis espacial)

- Estructura ideológica

\section{DISCUSIÓN HISTORIOGRÁFICA}

\section{DISCUSIÓN TEÓRICA}

\section{DISCUSIÓN METODOLóGICA}

El resultado de la clasificación (Figura 1) muestra de forma elocuente la tendencia seguida por la bibliografía reciente. Una más que significativa mayoría de la misma (69\%) cae dentro de la categoría general denominada "Descripciones". Dentro de la misma puede distinguirse entre publicaciones de informes de prospecciones o excavaciones (10.5\%) y trabajos descriptivos de un segmento muy específico del registro arqueológico: esculturas, mosaicos y pinturas, monumentos arquitectónicos, inscripciones y cerámica (60\% de todas las publicaciones).

La segunda categoría ("Interpretaciones de base epigráfico-literaria") supone un más modesto $27.17 \%$ del volumen total de trabajos publicados, mientras que en el apartado de "Interpretaciones arqueológicas", el panorama es desolador, ya que sólo un $2.53 \%$ de toda la bibliografía publicada en esas revistas durante los últimos quince años contiene trabajos de interpretación de datos arqueológicos. Mayor raquitismo padece aún el porcentaje de trabajos dedicados a propuestas y discusión teórico-metodológica y crítica historiográfica (1.44\%).

La tendencia expresada en este gráfico evoca inmediatamente un trabajo publicado por J. Alcina hace casi veinte años (Alcina, 1975a) en el que se denunciaba la situación de estancamiento positivista de la Arqueología española.

Se reconoce un masivo predominio de trabajos arqueográficos $(75.1 \%$ en la revisión de J.Alcina, $69.5 \%$ en el caso aquí tratado), sean memorias de trabajos de campo o puras descripciones de artefactos; dentro de este segundo grupo destaca el peso específico de las publicaciones de inscripciones $(22.1 \%)$ y esculturas $(13.7 \%)$. Las otras categorías de artefactos que más han centrado la actividad de los investigadores han sido mosaicos y pinturas, monedas y cerámica.

Tales trabajos descriptivos tienen la mayoría de la veces un propósito tipológico, para lo que se asume normalmente un enfoque exclusivamente morfológico/morfoscópico, renunciándose por regla general a tipologías tecnológicas o funcionales. Asimismo, la descripción no conlleva nunca una generalización estadística de la relevancia histórica de los ítems que se publican, sino que, antes bien, dada su naturaleza predominantemente artística, se prefiere siempre un comentario estilístico. La interpretación suele reducirse en la mayoría de los casos a la mención de casos particulares de analogía formal (paralelos), siempre desde una óptica ateórica y no cuantitativa. 
Siguiendo en este nivel arqueográfico, destaca la total ausencia de publicaciones de series de datos referentes a medio ambiente antiguo (geológicos, edafológicos, zoológicos o botánicos), recursos naturales (antracológicos o palinológicos) o demografía (paleoantropológicos y paleopatológicos), lo cual tiene su reflejo inmediato en la casi absoluta inexistencia (recordemos, un 2.53\%) de trabajos de interpretación arqueológica de las sociedades del periodo.

La sección "Interpretación del registro epigráfico-literario" fué individualizada al comprobarse que un considerable número de trabajos de interpretación se basaban únicamente en datos procedentes de autores antiguos y de inscripciones, mientras que los datos arqueológicos eran escasamente mencionados y siempre, en todo caso, con el propósito de reforzar postulados inducidos a partir de aquellos. Mucho más nutrida que la de interpretaciones arqueológicas, pero aún con porcentajes muy bajos, esta sección muestra una tendencia clara a ignorar problemas paleoambientales y demográficos $(1.8 \%$ del total), concentrándose en problemas socioeconómicos, ideológicos y de organización política y territorial (19.5\% del total). Apartados específicos serían el de conflictos bélicos (localización de sitios de batallas, campañas militares o invasiones), con un $2.17 \%$ y discusión de datos referentes a ciudades hispano-romanas específicas $(2.9 \%)$.

La discusión teórica no ha merecido ni un sólo trabajo en la vida de revistas que llevan hasta veinte años publicándose, de lo que parece inferirse que o bién ha existido un acuerdo absoluto entre los investigadores sobre los enfoques teóricos aplicables a la Historia Antigua de Andalucía, o bién este asunto es considerado completamente superfluo. El planteamiento de problemas metodológicos no ha merecido mucha mayor atención, ya que tan sólo en dos trabajos $(0.7 \%)$ se detecta interés, sea por explicitar los principios metodológicos de la investigación (Ponsich, 1991), sea por aportar una nueva vía de análisis (Ferreiro, 1985) de los datos ${ }^{2}$.

En síntesis, se dibuja un panorama arcaizante, similar al descrito por el profesor Alcina en 1975, que sugiere que dentro de la investigación actual de la Historia Antigua andaluza

"Si el concepto de Arqueología como ciencia auxiliar de la Historia ha sido desterrado como definición, efectivamente sigue siendo utilizada de esa manera para "ilustrar" el estudio histórico de las altas civilizaciones, en las que la abundancia de fuentes escritas hace, al parecer, inútil un análisis mas detallado y profundo de los materiales arqueológicos..." (Alcina, 1975a:52)

La hipervaloración de la interpretación del registro epigráfico-literario en detrimento de las evidencias empíricas arqueológicas y el gigantesco vacío de interpretaciones arqueológicas observable en la literatura son producto de las trayectorias históricas recientes propias de las dos grandes especialidades académicas que tienen como objeto la investigación del pasado clásico: la Arqueología clásica y la Historia Antigua.

\section{DISCUSIÓN}

De acuerdo con la evidencia mostrada por el gráfico descrito, en los medios académicos andaluces el aplastante peso de la tradición winckelmaniana parece haber propiciado que la Arqueología Clási-

2. El trabajo metodológico de $\mathrm{M}$. Ponsich sobre prospección y antigüedad no discute ninguno de los principios teóricos propuestos recientemente por varios prehistoriadores españoles en este campo (Ruiz Zapatero, 1988a, 1988b; Fernández, 1985; Ruiz Zapatero-Burillo, 1988; Fernández-Ruiz Zapatero, 1984). Por otra parte, el «modelo matemático» prometido en el título del trabajo de M. Ferreiro resulta ser finalmente el cálculo de dos medidas de tendencia central y desviación en varios conjuntos cerámicos. 


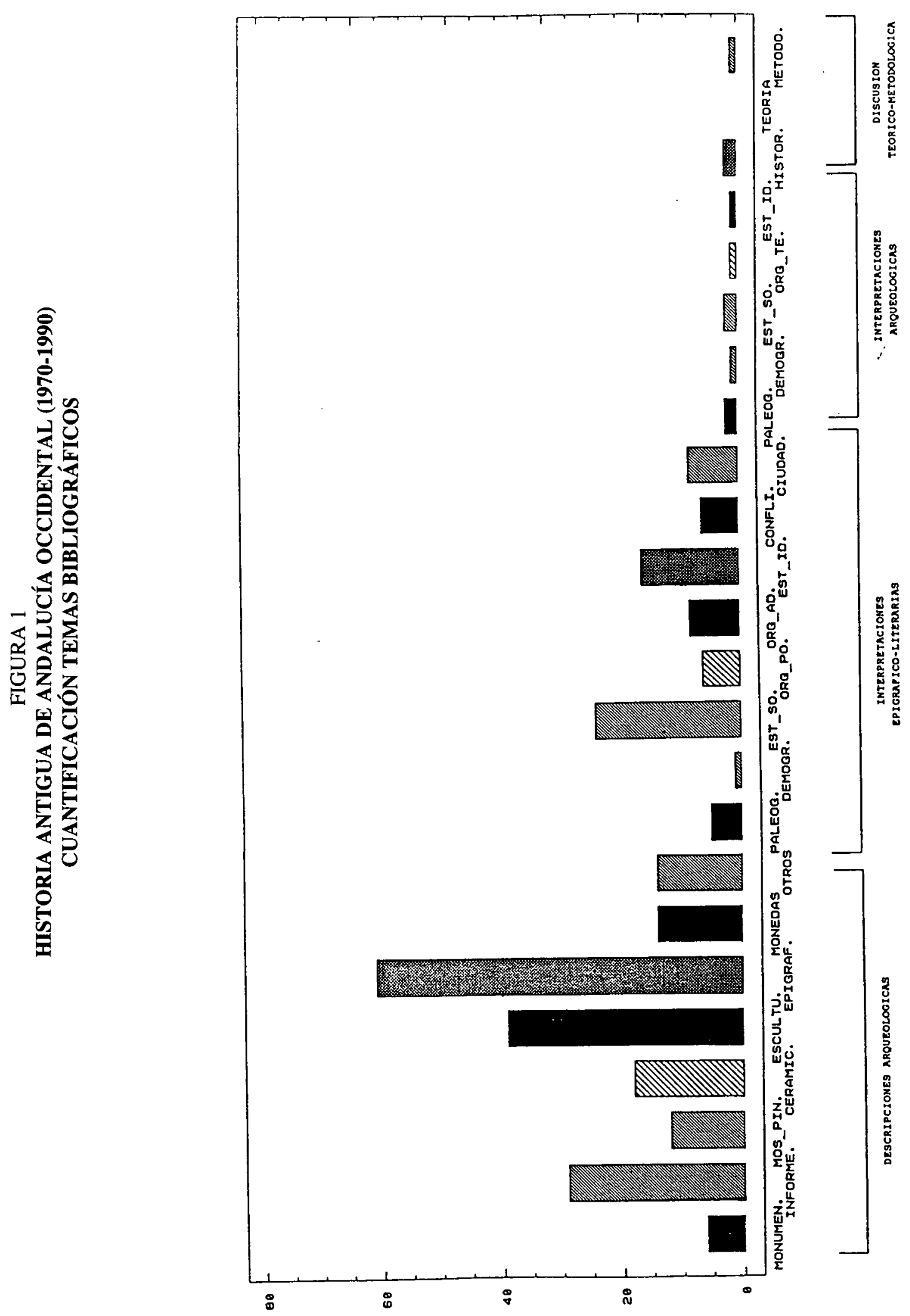


ca limite voluntariamente su propio campo de acción a las "bellas artes antiguas, situándose más próxima a la Historia del Arte que a la Antropología o la Historia (de las formaciones sociales primitivas/ precapitalistas). La ecuación Historia del Arte-Arqueología que emerge del examen cuantitativo de la bibliografía queda también reflejada en las escasas reflexiones o valoraciones conceptuales formuladas en la literatura en cuestión ${ }^{3}$ :

"Los hallazgos arqueológicos manifiestan siempre el interés del lugar y ponen de relieve la existencia de una faceta artística ${ }^{4}$ cuyo valor podemos apreciar en piezas como la que ahora nos ocupa..." (Luzón-León, 1971:234)

"De la importancia arqueológica de la ciudad de Orippo son elocuente testimonio sus amonedaciones, ruinas de edificios y piezas escultóricas, tan notables como el matrimonio sedente tomado de la mano, interpretado en un delicioso arte provincial..." (Bendala-Pellicer, 1977:321).

Así, en una primera instancia, los objetivos disciplinares generales quedan reducidos al nivel descriptivo-arqueográfico dentro de ese estrecho segmento del registro potencial que constituyen las manifestaciones artísticas. En una segunda instancia, tal reduccionismo se acentúa al ajustarse esta Arqueología Clásica al esquema epistemológico ultraconservador que ha caracterizado tradicionalmente a la Historiografía del Arte en Europa (Alcina, 1982), es decir, consideración del arte únicamente como elemento de goce estético, y consideración exclusiva de los productos artísticos de las clases sociales privilegiadas en cada momento histórico.

Tal esquema opera en las esferas téorica, metodológica, empírica e interpretativa, generando un flujo retroactivo constante y autorreproductivo: un marco conceptual estrecho delimita un estrecho segmento de evidencias relevantes, que a su vez generan postulados interpretativos de alcance limitado.

En la esfera metodológica, la insuperada fascinación conceptual por el objeto artístico se proyecta en forma de ignorancia o menoscabo hacia los contextos, hacia las estructuras territoriales (sociohistóricas y ecológicas) de distribución de la información arqueológica. La relevancia de un sitio para una intervención directa de recogida de datos no viene determinada por una reflexión explícita de su significación para la comprensión del sistema de poblamiento en que se enmarca, sino, directamente, por su monumentalidad. Subsecuentemente, se tiende por inercia a la práctica preferente de excavaciones de prestigio, es decir, exploración de las zonas nobles y monumentales de los sitios hispanorromanos, donde la probabilidad de localizar inscripciones y esculturas es considerada "a priori" mucho mas alta 5 . En algunos casos, se considera de forma explícita que la calidad del registro arqueológico asociado a un yacimiento está en función de dicha monumentalidad (Puertas-Rodríguez, 1979:105).

Inevitablemente, tal esquema de selección de evidencias propicia y facilita el sesgo del registro arqueológico disponible hacia los espacios ocupados por las clases sociales aristocráticas antiguas, de forma que la construcción de interpretaciones sobre la organización social circula en un recorrido cerrado de antemano. Ello se manifiesta en forma de desproporcionado interés por las discusiones centradas en torno a temas prosopográficos o de linaje aristocrático (Beltrán, 1991:178).

3. Puede aplicarse la afirmación realizada por V. Lull en otro contexto sobre el carácter extraordinariamente dificultoso de la tarea de

«...extraer «teoría» de la ingente bibliografía de corte empírico...» (Lull, 1991:231).

4. Todos los énfasis en citas textuales son nuestros.

5. Véase como ejemplo el caso de Itálica (Amores, 1986). 
El estudio preferente de las inscripciones monumentales y las esculturas nobles sesga la formulación de postulados relativos a la organización social antigua hacia las clases aristocráticas de tal manera, que en ocasiones llegará a confundirse inocentemente el supuesto esplendor de uno o varios linajes patricios con una incierta noción de bienestar de toda una comunidad:

"Prosopografía barbesulana: por fuentes epigráficas conocemos algunas de las familias más destacadas de Barbesula. Desde época flavia a los primeros severos destacan en barbesula los Fabii Fabiani, Vibii, Fulvii, Cervii, Cretii y los Aelii Severi. Algunas de estas familias se emparentan entre si, como es el caso de los Fabii Fabiani y los Fulvii (...) tales testimonios epigráficos nos hablan del esplendor de Barbesula en el siglo II, y de unas familias emparentadas entre si, que dominan los cargos municipales en una serie de ciudades de esta región de la Bética..." (Rodríguez, 1978:180)

Ocasionalmente, la particularización extrema del debate prosopográfico se expresa en forma de obsesión por la identificación individualizada de los personajes importantes del registro escultórico (Arce, 1976).

La interpretación de la estructura de relaciones sociales reflejada en el registro arqueológico de la antiguiedad queda por tanto, ya desde antes de su comienzo, irremediablemente reducida a esa clase social específica generadora de los bienes y productos de lujo que, como se ha visto, predominan ampliamente en la bibliografía (mosaicos, esculturas, pinturas, etc..). Las conflictivas relaciones entre el conjunto de clases sociales de la sociedad antigua, su naturaleza económica y su expresión política, no son objeto de interés, ni desde un punto de vista sincrónico ni desde un punto de vista diacrónico. Es decir, la discusión prosopográfica favorece un exacerbado particularismo genealógico y no una generalización cuantitativa basada en la formulación y contrastación de hipótesis sobre la expresión de las relaciones socioeconómicas del modo de producción esclavista dentro de cada medio histórico concreto.

En la esfera teórica, este esquema disciplinar alimenta (al tiempo que -naturalmente- es alimentado por) una concepción idealista y mentalista de la Historia abonada, como tantas otras veces, por estudios iconográficos, mitográfico-religiosos y simbólicos desenvueltos siempre en el contexto de las creencias e ideologías de las aristocracias antiguas, sin que como contrapartida se realice una lectura de tal iconografía tendente a valorarla en su contexto propagandístico y de clase ${ }^{6}$. El idealismo filosófico implícitamente emanante de esta producción bibliográfica tiene sólidas raices en la tradición académica precedente:

"Hay quienes atribuyen la evolución del hombre a factores externos al mismo, como pueden ser los cambios de clima y sus repercusiones en el medio ambiente; la abundancia o la escasez de caza, etc. Pero por encima de estos agentes, y sin restarles importancia, despunta la propia alma del hombre (...) en otro de los momentos más trascendentales de la Historia humana, el hombre pensó en liberarse de la servidumbre de la caza y de la recolección de frutos silvestres, y descubrió, como medio de conseguirlo, la domesticación de los animales y de las plantas." (Blanco, 1984:13)

Así, parece tenderse a una paradójica visión EMIC del pasado que ofrece una visión optimista del esplendor de las sociedades antiguas desde el propio marco ideológico de sus élites, tendencia que,

6. Veánse al respecto los interesantes trabajos de S. Eisenstadt (1966) o N. Hannestead (1986). 
como se verá a continuación, aparece reforzada por la utilización de informaciones fragmentarias procedentes de los productos literarios de tales élites.

Hablando en términos generales, este patrón de investigación tradicional está resultando ser extraordinariamente refractario a las transformaciones epistemológicas, teóricas y metodológicas que la Arqueología prehistórica española experimenta desde comienzos de los ochenta (Ruiz-Molinos-Hornos, 1986; Martínez, 1989; Vázquez-Risch, 1991; Alcina, 1991; Lull, 1991; Barandiarán-DelibesFernández, 1990; Díaz, 1993). Si después de las denuncias pioneras de J. Alcina (1975a, 1975b), la Arqueología prehistórica española ha iniciado un proceso irreversible de transformación que ha incluido a menudo sinceros esfuerzos de reflexión autocrítica, la Arqueología Clásica permanece ciertamente instalada en el limbo de lo que J.M. Vicent (1982) caracterizara como Paradigma anticientifista de la Arqueología española:

- Inexistencia de Programas de Investigación Interdisciplinares con objetivos sociales e históricos definidos.

- Antiteoricismo radical: inexistencia de declaración explícita de los presupuestos epistemológicos y teóricos que enmarcan cada investigación.

- Ingenuidad metodológica: confianza ilimitada en el razonamiento subjetivo.

Así, sorprendentemente se continúa ignorando la trascendente revolución teórica operada en la disciplina desde finales de los sesenta en tanto que, primero, debate sobre su propio estátus epistemológico (Salmon, 1982; Gibbon, 1986; Kelley-Hanen, 1990) y su posición respecto a disciplinas académicas afines (notablemente Antropología, Geografía e Historia) y, segundo, debate teórico interno sobre la naturaleza de la Historia y la Cultura a partir del registro arqueológico desde posicionamientos funcionalistas (Binford-Binford, 1968; Clarke, 1968; Binford, 1972, 1983a, 1983b, 1989; etc), marxistas (Bate, 1977, 1978; Montane, 1980; Spriggs, 1984; Mcguire, 1992; etc.) o estructuralistas/ "contextualistas" (Hodder, 1982, 1986; Shanks-Tilley, 1987a, 1987b; etc). La discusión teórica constituye, como se señalaba anteriormente, el más desfavorecido de los capítulos temáticos en que la muestra de bibliografía ha sido clasificada.

Por otra parte, la creciente sofisticación metodológica que se ha operado en el ámbito de la Arqueología prehistórica no ha tenido efecto alguno en las aproximaciones arqueológicas a la antigüedad que reflejan las revistas cuyos contenidos aquí son comentados. En primer lugar, la estrategia de selección de datos que evidencia el cuadro anterior (Figura 1) -implícitamente observable, ya que no expresa-, no incluye la integración de muestras de datos y técnicas de análisis paleoambientales en el diseño de la investigación. La contextualización del registro arqueológico/histórico en su correspondiente marco natural resulta de entrada imposible. Consecuentemente, las estrategias de análisis territorial del registro arqueológico (Hodder-Orton, 1976; Clarke, 1977; Hodder 1978a; Hietala, 1984; Grant, 1986; etc), así como de la versión automatizada más reciente de las mismas (Allen, 1991) no han sido objeto de aplicación ni tan siquiera de crítica. No se ha acometido una interpretación arqueológica de la organización del territorio en un contexto histórico en el que, precisamente, la acción integradora del estado romano genera un salto cualitativo fundamental en la antropización del paisaje, que proporciona indicadores empíricos propios de gran utilidad desde la perspectiva del análisis científico. La organización del aprovechamiento de recursos, el funcionamiento y evolución de redes de intercambio locales e internacionales, las relaciones entre las diferentes categorias de asentamientos y su evolución cronológica, no son objeto de análisis?.

7. Los estudios de arqueología romana del entorno académico inmediato, en cambio, sí están incorporando paulatinamente estas aproximaciones (Ruiz-Molinos, 1986; Cerrillo, 1988; Choclán-Castro, 1988; etc). 
La historiografía también ha obviado la posibilidad de analizar las relaciones sociales desde un marco de referencia no cerrado y particular sino abierto y general como el constituido por el registro arqueológico funerario, como también se ha obviado el estudio paleoantropológico (demografía, nutrición, mortalidad, etc.) del mismo. Por otra parte, la crucial aparición y desarrollo del razonamiento arqueológico estadístico, aplicado a todos los niveles del análisis arqueológico (Clarke, 1968; Mueller, 1975; Doranz-Hodson, 1975; Borillo, 1977; Bietti, 1979, 1982; Orton, 1980; Carr, 1985; Aldenderfer, 1987; Shennan, 1988; etc), sea en la construcción de taxonomias numéricas, sea en la exploración de las relaciones entre variables múltiples, y la consiguiente apertura posterior del mismo a las tecnologías de la información, no parece haber sido asumida como conjunto de herramientas destinadas a racionalizar los sistemas de inferencia arqueológicos, con lo cual se perpetúa el enfoque epistemológico de corte subjetivista.

Finalmente, la literatura adolece de una falta absoluta de reflexión sobre el impacto social y público de la Arqueología y sobre la naturaleza específica de la gestión del Patrimonio arqueológico antiguo a todos los niveles (catalogación, protección y divulgación) en una etapa en la que precisamente, por primera vez en la historia de la Arqueología de nuestra comunidad se han abierto expectativas de desarrollo profesional dentro de la disciplina.

Por su parte, la Historia Antigua, reducida cada vez más a una práctica exclusivamente académica, no parece haber sabido responder al doble desafío planteado en la pasada década:

- Por una parte, ha quedado absolutamente excluida de los nuevos sistemas de gestión, investigación y protección del patrimonio histórico que se desarrollan en nuestra comunidad autónoma desde mediados de los ochenta.

- Por otra (y en esto comparte andadura con la Arqueología Clásica), no sólo ha ignorado la evolución de la disciplina arqueológica mencionada arriba, sino que además, salvo loables excepciones, ha institucionalizado la negación pura de la evidencia misma de que el registro empírico con el que cuenta para construir el proceso dialéctico de formulación y contrastación de modelos teóricos es abrumadoramente arqueológico. Desde esta óptica, la Arqueología parece seguir siendo presentada como una

“...ciencia casi inútil para lo que no sea cultura material” (Presedo, 1986:283)

La causas de esta preferencia por la selección de datos tomados del registro epigráfico-literario, en detrimento del arqueológico, para la construcción de interpretaciones históricas de la antigüedad no quedan explicitadas en la bibliografía. La devaluación de los datos arqueológicos supone la pérdida de una fuente no sujeto de ideologización previa (como es el caso del texto), poseedora de un potencial informativo susceptible de introducir nuevos argumentos de estudio así como matizar o incluso remodelar completamente el conocimiento acumulado de este periodo histórico.

Evidentemente no se trata de disminuir mecánicamente el valor de la información proporcionada por las fuentes literarias, que contienen un alto indice de fiabilidad para algunos temas, pero no se debe olvidar que en su mayoría fueron generadas por autores que desarrollaron su trabajo en el contexto ideológico y social de las élites sociales antiguas, y que han legado una visión de la historia ligada a intereses de clase o de estado (Gabba, 1980, 1974; Arce, 1989; Starr, 1965; Finley, 1985; Clavel, 1974a; Fontana, 1990) que precisa por tanto de un esfuerzo de contextualización y crítica previo a su inclusión en el discurso científico. 
Los problemas historiográficos a que está sujeta la utilización de datos tomados del registro literario antiguo han sido discutidos por investigadores españoles en relación con la protohistoria peninsular (González, 1986a; García, 1979; Bermejo, 1983) o con determinados aspectos de la romanización (Espinosa, 1984; Ramírez, 1985; Arce, 1989), pero si por algo se caracteriza la muestra de artículos descrita en el epígrafe anterior, es precisamente por su total acriticismo al respecto. Al igual que los trabajos de discusión teórico-metodológica arqueológica resultaban cuantitativamente despreciables, cabe destacar que no ha sido registrado ningún artículo referido a esta cuestión entre las revistas que hemos seleccionado, a pesar del masivo uso que los encuadrados en el apartado de "Interpretaciones epigráfico-literarias" hacen de textos de autores antiguos que escribieron sobre la Bética hispanoromana, notablemente Estrabón y Plinio, y de los problemas que su utilización como fuente de datos históricos presenta (Arce, 1989; Keay, 1992).

A este respecto, G. Bravo ha propuesto como imprescindible la realización de una descodificación de las fuentes indirectas para que

“...los testimonios puedan ser homologables y comparables.” (Bravo, 1985:40)

clasificando por ejemplo las fuentes literarias de acuerdo con diferentes criterios como pueden ser la riqueza personal o la ideología de sus autores:

"Es presumible que la formulación de hipótesis en Historia Antigua sea incluso más frecuente que en ninguna otra disciplina histórica para resolver los problemas que plantea una documentación lacónica (...) Ello exige a menudo completar la crítica externa (autenticidad) e interna (fiabilidad) con la prueba de concurrencia de fuentes de diversa naturaleza; si la convergencia es total no se suele dudar de la fiabilidad del testimonio. Pero en algunos casos, en que la información proviene unilateralmente de un mismo tipo de fuente es preciso preguntar al texto no qué dice sino qué oculta e incluso qué pretende decir u ocultar" (Bravo, 1985).

Sin embargo, si por una parte la Arqueología Clásica reduce sus objetivos disciplinares a la descripción de una cultura material antigua reducida al arte noble (mosaicos, esculturas, joyas, etc) y al artesanado de lujo y por otra la Historia Antigua ignora con su acriticismo la plausible posibilidad de que la visión de la antigüedad que utiliza como fuente de sus postulados científicos esté razonablemente tergiversada, se constata entonces cómo para en la reconstrucción del periodo histórico que se ha venido a denominar antiguo, se

“... a favorisé l"élaboration d'une conception optimiste, dominante, d'un passé riche en civilisations brillantes, matérialisées dans des vestiges souvent remarquables qu'on leur a assignée dans les fondements de la civilisation occidentale, passé qui de plus se révèle dans la transparence du témoignage unilatéral de l'élite dirigente, justifiant sa propre pratique de classe" (Clavel-Favory, 1977:98).

Otra de las características del conjunto de trabajos examinados es la extraordinaria importancia concedida al registro epigráfico. La publicación de inscripciones per se supone casi una cuarta parte (22\%) del total de artículos, mientras que, como ya se ha dicho, los datos epigráficos suponen el segundo gran soporte de la inmensa mayoría de las interpretaciones realizadas.

De nuevo, sin embargo, se detecta una falta absoluta de discusión crítica de la naturaleza de los datos aportados por este segmento del registro: por una parte, muchas de las inscripciones publicadas están descontextualizadas de toda referencia arqueológica, sea estratigráfica, sea micro o semimicro espacial, ¿carece este asunto de relevancia? Por otra ¿producían todas las clases sociales de la Bética 
hispano-romana la misma cantidad de inscripciones monumentales? ¿Existe riesgo de que la investigación de la sociedad antigua se encasille definitivamente hacia cuestiones de linaje? La falta de reflexión en torno a tales posibles problemas inherentes al registro epigráfico resulta tanto más incomprensible en cuanto que, como se veía anteriormente, sostiene por la base el entramado interpretativo generado por la historiografía reciente: véase una excelente discusión de estas cuestiones en (Samson, 1989).

La clásica propuesta antiteórica de acumulación de datos hasta el infinito, y la ausencia de esfuerzos de reconstrucción histórica, parecen herencia de una historiografía tradicional que considera irresponsable la elaboración de hipótesis o interpretaciones históricas sin contar primero con la totalidad de los datos posibles, pretensión evidentemente inalcanzable (Keller-Clavel-Millote, 1976; Plá, 1982) y, en todo caso, absurda. Esta ausencia de debate teórico sobre la naturaleza de la Historia en la muestra de trabajos publicados debe relacionarse con la inexistencia de un debate teórico-metodológico enriquecedor como el que ha convulsionado a la Arqueología española (fundamentalmente de épocas pre y proto-históricas) después del impacto de las propuestas procesualistas a principios de los ochenta.

Fuera de nuestras fronteras, desde mediados de los setenta, una avanzadilla progresista planteó la necesidad de una renovación metodológica y teórica en los estudios de Historia Antigua, (ClavelFavory, 1977; Freedman-De Laet-Barraclough, 1981; Favory, 1981; Humphreys, 1989; CapogrosiGiardina-Schiavone, 1978), producto de la cual serían los trabajos de los grupos de Besançon y Pisa, que iniciaron una andadura de fecundas investigaciones sobre las formaciones sociales esclavistas (VVAA, 1972, 1974, 1976). A partir de entonces destacan los trabajos de J. Annequin (1982; 1983; 1985a; 1985b; 1991), A. Carandini $(1979 ; 1983 ; 1986$; 1989; Carandini-Settis, 1979), M. ClavelLévêque $\left(1974 b ; 1974 c ; 1975 ; 1976 ; 1977 a ; 1977 b\right.$; etc), o Ch. Parain $(1965 ; 1977)^{8}$. La reacción primitivista contra esta escuela de corte modernista se gestó en Cambridge con M.I. Finley como máximo exponente (Finley, 1975).

Las diferencias entre ambas escuelas no se establecen sólo en un plano conceptual, sino también metodológico. Los primitivistas circunscriben mayoritariamente los estudios de Historia Antigua al marco de las culturas griega y romana obviando las periféricas, que no participaban del genio de las anteriores. La sobrevaloración de ambas culturas impide desarrollar una aproximación antropólogica, al considerar la imposibilidad de establecer analogías, por el caracter único e irrepetible de las mismas. Por el contrario, la escuela materialista concibe en su conjunto el mundo antiguo destacando sobre todo las mutaciones sufridas por el mismo a lo largo de su existencia, desmitificando su particularidad y enlazándolo al proceso histórico general. De este modo no solo es posible establecer analogías antropológicas, sino que es indispensable en tanto que se persigue la formulación de leyes generales de desarrollo histórico.

La crítica a estas escuelas materialistas se ha centrado naturalmente en su exceso de teorización y falta de aportaciones empíricas de carácter enciclopédico-erudita

“...en ce qui concerne l'Empire romain, les marxistes français apparaissent d'abord soucieux de réflexion théorique et n'ont pas proposé d'ouvrage de grande ampleur" (Jacques-Scheid, 1990).

8. En España este proceso tuvo una breve repercusión materializada en la celebración de unos coloquios de Historia Antigua en Oviedo iniciados en 1977 que pretendieron, generar las bases epistemológicas de una nueva investigación de la Historia Antigua; tal ensayo fué aparentemente ignorado en Andalucia Occidental en tanto que el esfuerzo de generalización no sustituyó a la particularización descriptiva basada en los pseudopresupuestos tradicionales. 
Si consecuentemente con esta visión tradicional de la disciplina, muchos investigadores (Nicolet, 1988; Finley, 1975; Jacques-Scheid, 1990) siguen negando la necesidad de reformar los sistemas de reconstrucción histórica con la aplicación a los estudios de la antigüedad de concepciones antropológicas y un debate teórico profundo y constante, desgraciadamente parece que es a esta visión a la que se han adscrito explícita o implícitamente la mayor parte de los investigadores de la Historia Antigua en Andalucía occidental, a juzgar por el bajo estátus que conceden a la teorización; es decir

“...apenas se plantean cuestiones que desborden su campo particular de investigación...” (Bravo, 1985:27).

no existiendo contrastación en el sentido de

“...legitimar los resultados de forma similar a como proceden otras disciplinas del espectro científico, mediante verificación de hipótesis, validación de teorías u otras operaciones formales que la crítica historiográfica no dudaría en calificar de teóricas o metodológicas." (Bravo, 1985:27).

La crítica a la ausencia de conceptualización por una parte y a la obsesión por la acumulación de datos por otra, formulada en los años setenta contra la investigación histórica en general

"No cabe duda (...) de que somos absolutamente contrarios al empirismo teórico que caracteriza a las ciencias sociales y a la historia en sus versiones académicas. En estas disciplinas científicas el conocimiento queda reducido a los hechos "dados" (...). En consecuencia, la ciencia comienza con la cuidadosa observación y recolección de hechos y termina con la correlación de estos. (...) Desafortunadamente para tales actitudes, para el conocimiento los hechos no son nunca "dados"; siempre son producidos. Pretender que no sea así y concebir que hay ciertos elementos del conocimiento que se dan en la realidad equivale a negar el papel central de la práctica científica, tanto de la experimentación como de la construcción y la argumentación teóricas explícitas en la producción del conocimiento cientifico." (Hindess-Hirst, 1979:6).

y a la arqueológica en particular

"Pour l'archéologue traditionel, l'archéologie, science du passé, est surtout une science des faits, des objets réels. (...) Aussi le champ de la connaissance et-il infini (on trouvera toujors de nouveaux objets) et indéfiní (on ne sait pas ce que représente une trouvaille). Une ville, un monument, un objet isolé ne sont que les témoignages d'une culture." (Cleziou-Demoule-Schnapp, 1973:35).

puede trasladarse en los mismos términos a las investigaciones publicadas hasta el momento en las revistas seleccionadas para este trabajo.

Ahora bién, ¿es la situación descrita del todo exclusiva de la investigación de la Historia Antigua en Andalucía, o por el contrario ésta simplemente refleja una realidad más amplia, de alcance nacional? Para intentar avanzar una respuesta orientativa a esta cuestión, y sin ánimo alguno de exhaustividad, se ha realizado una contrastación de los resultados obtenidos y previamente comentados, con la cuantificación temática de la bibliografía de dos revistas de alcance nacional asimismo especializadas en Historia Antigua y Arqueología Clásica:

- Gerión. Revista de Historia Antigua de la Universidad Complutense de Madrid (1983-1990).

- Archivo Español de Arqueología. Revista de Arqueología e Historia Antigua del Instituto Rodrigo Caro del CSIC (1970-1990)

9. Dada la antigüedad de esta publicación, hemos tomado nuestra muestra sólamente a partir de 1970, fecha del inicio de la publicación de la revista más antigua de todas las examinadas en la primera muestra, Habis. 
Comparando los porcentajes obtenidos anteriormente con los resultantes de la clasificación de los 267 trabajos consultados de ambas publicaciones (Figura 2) se obtiene un cuadro en el que se constata rápidamente la similitud de la distribución de proporciones (Figura 3). De nuevo, la mayor parte de la bibliografía (58.1\%) cae en el apartado de "descripciones arqueológicas", aunque en este caso tal predominio sea algo menor que en la muestra anterior (69.5\%). De nuevo son las descripciones de esculturas, inscripciones, mosaicos, pinturas y edificios monumentales las que predominan en este apartado (44.4\% del total, frente al $42.3 \%$ en la muestra de revistas de Andalucía Occidental). Comparativamente, los informes de excavaciones y prospecciones siguen ofreciendo porcentajes muy inferiores $(4.4 \%$, es decir, también bastante por debajo del $9.5 \%$ de la muestra de revistas andaluzas).

La categoría de "Interpretaciones de base epigráfico-literaria" supone en conjunto un 33.3\% (algo superior al $27.1 \%$ registrado en la otra muestra), resultando de nuevo poco significativos los trabajos dedicados a cuestiones de medio ambiente o recursos naturales y demografía. La mayoría de las publicaciones de esta sección se orientan a la discusión de la estructura socieconómica y política $(25.4 \%)$ tal y como ocurría en la muestra anterior. El porcentaje de artículos que tienen como objeto la discusión de asuntos bélicos es ligeramente más alto que en la muestra anterior ( 3.7 frente a 2.1 , mientras que el problema de la localización de ciudades y comentario de los datos existentes presenta idéntico porcentaje en ambas muestras (2.9).

Por su parte, las "Interpretaciones arqueológicas" de nuevo asumen un porcentaje prácticamente despreciable (3.7\%). Es de reseñar que, dentro de la segunda muestra, el $70 \%$ de las escasísimas interpretaciones de datos arqueológicos existentes se refieren a problemas mitológicos y religiosos. Ningún trabajo ha sido dedicado a estudio social del registro funerario o análisis territorial de datos espaciales.

Finalmente, en el apartado de discusión teórico-metodológica tampoco se aprecian grandes cambios. De los 5 trabajos dedicados a teoría histórica o arqueológica (1.8\% del total frente al $0.0 \%$ anterior) al menos dos son firmados por prehistoriadores (Lull-Picazo, 1989; Vila-Estévez, 1989), uno por un historiador extranjero (Alföldy, 1983), mientras que de las dos propuestas metodológicas registradas, una de ellas es firmada por paleolitistas (Mora-Carbonell, 1990).

En general, ambas publicaciones tomadas en conjunto comparten con las ocho analizadas previamente las principales características ya discutidas, es decir, antiteoricismo, nivel arqueográfico artístico generalizado, primacía de las interpretaciones históricas epigráfico-literarias sobre las arqueológicas, ausencia de propuestas metodológicas renovadoras. Las (una vez más escasas) declaraciones programáticas aparecidas en esta muestra de trabajos emanan de una concepción de la Arqueología Clásica no muy distinta a la que veíamos anteriormente:

"Entre los numerosos vestigios arqueológicos de época romana que posee la provincia de Jaén, tienen un lugar preferente los monumentos escultóricos. Este hecho, que pudiera parecer obvio a los ojos del investigador [sic], adquiere especial interés cuando se profundiza en su estudio y se hace evidente la complejidad de los motivos iconográficos, estilísticos y estéticos que aparecen en ellos representados." (Baena, 1984:47).

Nótese que L. Baena considera obvio el hecho de que las esculturas representan la categoría más importante del registro arqueológico de época romana en Jaén, siendo precisamente el valor iconográfico y estilístico de las mismas el que así lo demuestra; Arqueología e Historia del Arte (de la nobleza antigua) siguen siendo equivalentes. 

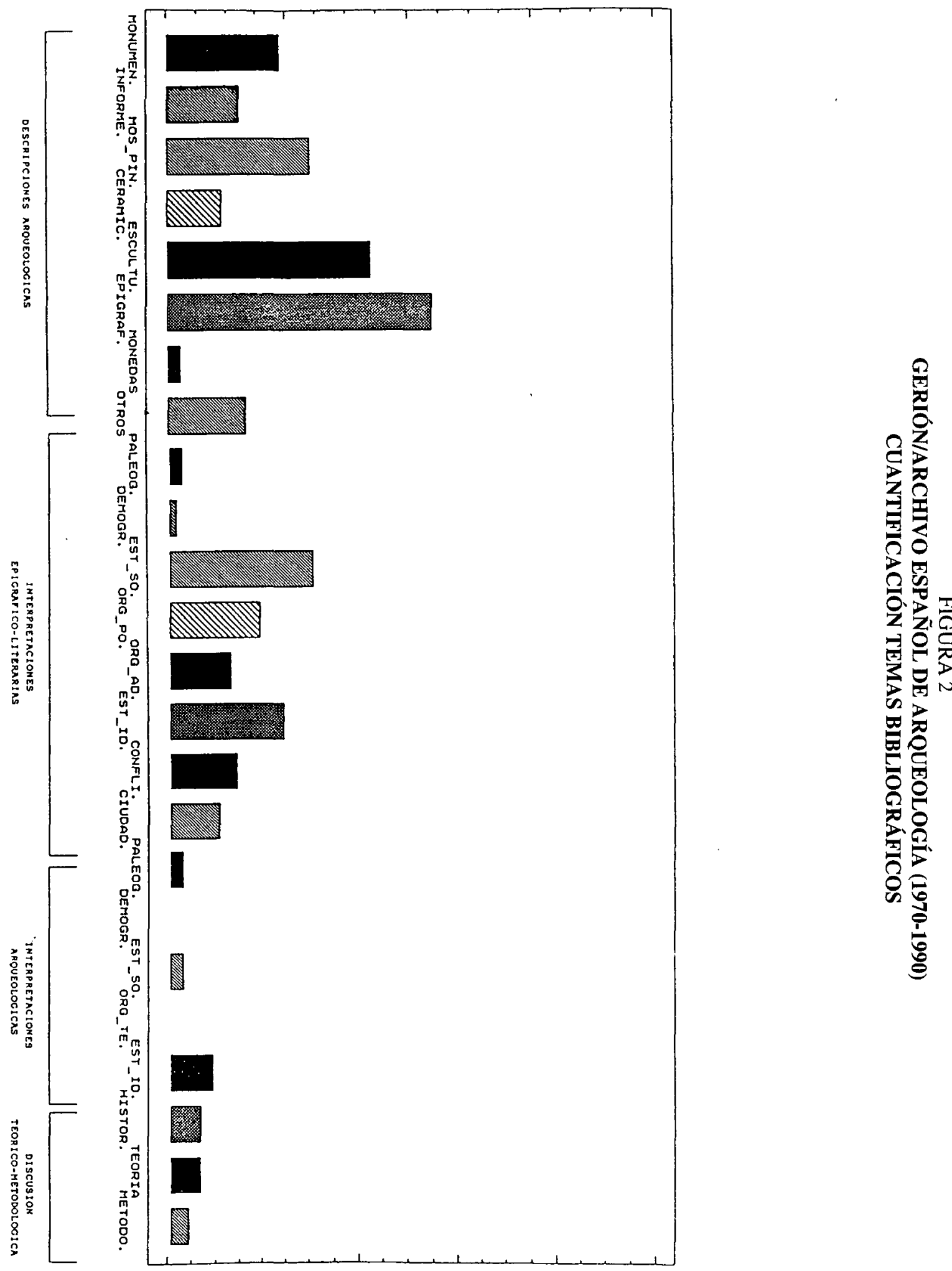
FIGURA 3

DISTRIBUCIÓN TEMÁTICA DE LA BIBLIOGRAFÍA EN AMBAS MUESTRAS:

TABLAS COMPARATIVAS

\begin{tabular}{|c|c|c|}
\hline BLOQUE TEMÁTICO & $\begin{array}{c}\text { MUESTRA A } \\
\text { (ANDALUCÍA OCC.) }\end{array}$ & $\begin{array}{c}\text { MUESTRA B } \\
\text { (GERION/AESPA) }\end{array}$ \\
\hline $\begin{array}{l}\text { Esculturas } \\
\text { Inscripciones } \\
\text { Mosaicos/Pinturas } \\
\text { y Monumentos } \\
\text { Informes Prospecciones/ } \\
\text { Excavaciones } \\
\text { Total "Descripciones } \\
\text { arqueologicas" }\end{array}$ & $\begin{array}{r}13.7 \% \\
22.1 \% \\
6.5 \% \\
9.5 \% \\
69.5 \%\end{array}$ & $\begin{array}{l}12.7 \% \\
16.4 \% \\
15.3 \% \\
4.5 \% \\
58.1 \%\end{array}$ \\
\hline $\begin{array}{l}\text { Paleoambiente/ } \\
\text { Demografía } \\
\text { Estructura socioec./ } \\
\text { Org. política y } \\
\text { territorial/Est. ideológica } \\
\text { Conflictos bélicos } \\
\text { Ciudades } \\
\text { Total "Interpretaciones } \\
\text { epigráfico/literarias }\end{array}$ & $\begin{array}{r}19.5 \% \\
2.1 \% \\
2.9 \% \\
\\
27.1 \%\end{array}$ & $\begin{array}{r}25.4 \% \\
3.7 \% \\
2.9 \% \\
33.3 \%\end{array}$ \\
\hline $\begin{array}{l}\text { Total "Interpretaciones } \\
\text { arqueológicas" }\end{array}$ & $2.5 \%$ & $3.7 \%$ \\
\hline Total Teoría & $0.0 \%$ & $1.8 \%$ \\
\hline Total Metodología & $0.7 \%$ & $1.1 \%$ \\
\hline
\end{tabular}

\begin{tabular}{|cccc|}
\hline TABLA 1 & $\begin{array}{c}\text { DESCRIPCIONES } \\
\text { ARQUEOLÓGICAS }\end{array}$ & \multicolumn{2}{c|}{$\begin{array}{c}\text { INTERPRETACIONES } \\
\text { ARQUEOLÓGICAS }\end{array}$} \\
\hline MUESTRA A & 192 & 7 & 199 \\
MUESTRA B & 155 & 10 & 165 \\
\hline & 347 & 17 & 364 \\
\hline
\end{tabular}

\begin{tabular}{|cccc|}
\hline $\begin{array}{c}\text { TABLA } 2 \\
\text { INTERPRETACIONES } \\
\text { ARQUEOLÓGICAS }\end{array}$ & $\begin{array}{c}\text { INTERPRETACIONES } \\
\text { EPIGRAF. 4T }\end{array}$ \\
\hline MUESTRA A & 7 & 75 & 82 \\
MUESTRA B & 10 & 89 & 99 \\
\hline & 17 & 164 & 181 \\
\hline
\end{tabular}

\begin{tabular}{|lccc|}
\hline TABLA 3 & TEORÍA & \multicolumn{2}{c|}{ METODOLOGÍA } \\
\hline MUESTRA A & 0 & 2 & 2 \\
MUESTRA B & 5 & 3 & 8 \\
\hline & 5 & 5 & 10 \\
\hline
\end{tabular}


Por otra parte, que las reconstrucciones históricas han de ser formuladas a partir de la lectura de inscripciones y textos clásicos es asimismo considerado autoevidente o lógico por algunos autores:

"Pero independientemente del problema de su localización [Municipium Florentinum Iliberritanum], al que se ha venido prestando atención exclusiva, es factible analizar y estudiar, en base a la documentación literaria, y sobre todo epigráfica, diversos aspectos de la sociedad, economía, religión, instituciones, etc." (Pastor, 1983:151).

La contrastación realizada a partir de los datos tomados de estas dos revistas sugiere por tanto que el patrón de investigación definido en el estudio de la Historia Antigua en Andalucía Occidental no es único ni singular, sino que reflejan tan sólo los rasgos principales de tal disciplina a nivel nacional ${ }^{10}$.

\section{CONCLUSIÓN}

La necesidad misma de reclamar (ante la constatada inexistencia de autocriticismo) como urgente un debate teórico y metodológico (a un nivel básico ya ampliamente superado en, por ejemplo, la Arqueología prehistórica o la Antropología) sugiere que la investigación de la Historia Antigua y de la Arqueología Clásica en Andalucía Occidental se encuentra estancada en un paradigma insatisfactorio.

La similar situación de obsolescencia epistemológica institucionalizada de las arqueologías clásicas y de las historias antiguas de los medios académicos anglosajones ha generado rẹacciones autocríticas individualizadas durante los ochenta (Dyson, 1981; 1989; 1993; Snodgrass, 1985) que paulatinamente se van generalizando en forma de debate (Scott, 1993). En España, recientes trabajos han apuntado este desfase en el contexto nacional,

“...una excesiva y frecuentemente mal asimilada dependencia de la arqueología germana es en mi opinión la responsable de este ultrapositivismo arqueológico, mientras que se continuan ignorando gran parte de los planteamientos teóricos y metodólogicos que inspiran actualmente la investigación arqueológica en otros muchos lugares." (González, 1986b:131).

“...desde nuestro punto de vista parece evidente el retraso de la investigación sobre Historia Antigua que padecemos en nuestro país." (Remesal et alii, 1991:220).

“...los más antiguos y graves inconvenientes de la investigación en Historia Antigua en este país: su localismo corto, su patrioterismo barato, su incorregible ignorancia y su presunción sin límites totalmente acientifica (o mejor, anticientifica)." (Arce, 1985:452).

10. Ello se constata rápidamente por la vía estadística, si aplicamos una sencilla prueba de significación a las tablas de contingencia mostradas en la Figura 3. Realizando para las dos primeras la prueba de Chi-Cuadrado (en la tercera se rompe uno de los supuestos teóricos del test, cual es que no debe darse ninguna frecuencia esperada menor que cinco) para clasificaciones cruzadas, situando el nivel de significación en 0.05 (95\% de probabilidades de ser correctos en la estimación), para la primera tabla obtenemos un valor de 1.25 , y para la segunda de 0.12 , es decir, ambos menores que el valor tabulado de Chi para dos Grados de Libertad (3.84); aceptando $\mathrm{H}_{0}$ no hay diferencia entre ambas muestras en términos de trabajos publicados bajo las categorias referidas. Por otra parte, si para la Tabla 3 calculamos el Test Exacto de Fisher, también situando Alfa en 0.05 , el resultado es 2.22 , con lo cual tampoco se alcanza el umbral de rechazo de $\mathrm{H}_{0}$, de lo que debemos seguir que ambas muestras tampoco son diferentes en términos de abundancia de literatura teórica y metodológica. 
"El desarrollo y la calidad científica de la Historia Antigua en España merecen, genéricamente, el calificativo de deficientes." (Arce-Plácido, 1990:19).

pero en lo que se refiere a la muestra de trabajos aquí utilizada, tales trabajos han tenido escaso eco.

Parece claro que la observada ausencia de construcciones teóricas fundamentadas en análisis complejos de evidencias empíricas de calidad, no puede ser compensada por la inocente propuesta factualista y positivista de presentar básicamente hechos (i.e. datos) ${ }^{11}$ que, por una parte, ignora que, en el contexto de las ciencias sociales, los hechos en general no existen ni se observan de forma aleatoria o pura sino que son recogidos (y adquieren sentido) en el marco de sistemas de observación, selección y procesamiento asociados a criterios teóricos e ideológicos ineludibles y preexistente (Katuzian, 1985), mientras que, por otra parte, facilita una visión del pasado optimista centrada en las clases aristocráticas y sus productos artísticos y literarios.

La alternativa de un estudio de la antigüedad andaluza fundamentado en el análisis de evidencias empíricas cuantificables procedentes de todos los segmentos del registro arqueológico, y de un uso crítico, selectivo y contextualizado de las fuentes epigráfico-literarias, aparece pues como una necesidad. La mejor aproximación posible a la antiguiedad no es necesariamente aquella que se presenta en forma de inducciones procedentes de los datos de estas últimas fuentes, acéfalas de marcos teóricos, ni existen razones objetivas para que la Arqueología Clásica se mantenga circunscrita al campo de la Historia del Arte antiguo ignorando lo mejor de veinte años de transformaciones en la Arqueología prehistórica.

Partiendo de la aceptación de que la investigación en Arqueología o Historia persigue la formulación de generalizaciones probabilístico/estadísticas que expliquen la variabilidad de los datos disponibles, parece altamente improbable que los datos útiles aportados por el registro epigráfico -por no decir el literario- alcancen alguna vez un volumen suficiente como para permitir evaluaciones estadísticas complejas (i.e. contrastaciones), o como para permitir que éstas se superpongan sucesivamente permitiendo la formulación de dichas generalizaciones. En otras palabras, si los textos pueden sugerir hipótesis, contribuir a su formulación, nunca aportarán recursos empíricos suficientes como para efectuar una contrastación estadística de las mismas, al menos en lo que a Historia Antigua de España se refiere.

Una auténtica lectura arqueológica de la antiguiedad, planteada con todo el ánimo de ser utópicos, requerirá de numerosas transformaciones en las actuales estructuras académicas y científicas y de una evolución conceptual considerable. La delimitación académica Arqueología Clásica vs. Historia Antigua existente en numerosas facultades no se justifica por el objeto del conocimiento que promueven, idéntico para ambas, sino por la existencia de tradiciones disciplinares diferentes (Historia del Arte vs. Filología); la incomunicación y aislamiento mutuos resultan tanto más incomprensibles en tanto que dificultan el tránsito hacia la interdisciplinareidad.

\footnotetext{
11. Típica de la historiografía tradicional de la Historia:

"...aquellos historiadores que hacen un enfoque tradicionalista sostienen que los hechos fueron Historia y que no hay nada que sustituya la erudición y la búsqueda de ese pequeño hecho verdadero.»(Plá, 1982:17).

No han faltado intentos de sistematización de esta postura en la literatura arqueológica reciente. La propuesta de que:

"...the establishment of facts is the archaeologist's proper role and mission.» (Courbin, 1988:132).

tiene su equivalente castizo en nuestra propia historiografía arqueológica:

"... [el arqueólogol sobre todo ha de sentirse esclavo de la limitación evidente de los documentos que tiene en sus manos (...) al servicio de esta verdad básica no dejaremos jamás de oponernos y de salir al camino de algunos teorizantes que andan entre nosotros.» (AImagro, 1960:83).
} 
Una investigación operativa de la Antigüedad y la formación de técnicos de gestión del patrimonio arqueológico e histórico conllevan una serie de necesidades propias a las que por el momento la Arqueología Clásica y la Historia Antigua dan la espalda al unísono. Como consecuencia directa del predominio en Andalucía Occidental de una anacrónica concepción de Arqueología Clásica, el estudiante universitario que se aproxime a la historia antigua se encuentra enfrentado a una serie de asignaturas de iconografía artística rellenadas con acrítica perspectiva anticuarista y no a una formación teórica y metodológica apropiada para el análisis de la sociedad hispano-romana a partir de los datos arqueológicos o para la formación de equipos de gestión y protección del patrimonio antiguo de nuestra comunidad autónoma. La inadecuación de la mayoría de los planes de estudio universitarios de nuestro país para formar técnicos y profesionales en este emergente campo (Ruiz Zapatero, 1991; Hernando, 1992; Criado, 1988; Barandiarán-Delibes-Fernández Miranda, 1990), no debe ser excusa para perpetuar una situación obsoleta.

Por la parte que corresponde a la Historia Antigua, una vez perdida la pista de la evolución del debate arqueológico reciente, se ha negado la entrada de cualquier tipo de formación arqueológica (teórica y metodológica) a los estudiantes de la especialidad (Arce-Plácido, 1990; Gómez, 1988), enfatizando la formación literaria hasta lo irracional, teniendo en cuenta que se inculca un enfoque exclusivamente filológico-literario y no histórico-crítico, fundamental a la hora de utilizar unas fuentes escritas voluntariamente tendenciosas en muchos casos.

¿Contribuye tal visión de la docencia universitaria a transformar la situación descrita? No lo parece realmente. La realidad es que en el caso particular de los licenciados en ambas especialidades por las universidades de Andalucía Occidental, la carencia de una formación arqueológica práctica, social, científica y profesionalmente aplicable a la gestión del patrimonio histórico, no resulta ser producto tan sólo de las limitaciones de infraestructura, sino también de la concepción epistemológica implícitamente predominante en la disciplina. Ello supone una hipoteca de futuro, una buena parte de cuya responsabiblidad recae sin duda en las instituciones universitarias.

\section{BIBLIOGRAFÍA}

ALCINA, J. (1975a): "La Arqueología antropológica en España:situación actual y perspectivas" En JIMÉNEZ, A. (ed): Actas de la Primera Reunión de antropólogos españoles (Sevilla,1973). Sevilla 1975b: "Arqueología y Antropología" La Antropología en España. Vol 42 num 97. Madrid 1982: Arte y Antropología. Madrid. Alianza editorial.

1991: "La Arqueología en España: una revisión crítica de sus planteamientos teóricos." Trabajos de Prehistoria num 48. Madrid. CSIC

ALDENDERFER, M.S. (1987): Quantitative research in Archaeology. Progress and prospects. Beverly Sage Publications

ALFÖLDY, G. (1983): "La Historia Antigua y la investigación del fenómeno histórico." Gerión num 1. Madrid

ALLEN, K.M. et alii (eds) (1990): Interpreting space: GIS and Archaeology. London. Taylor and Francis

ALMAGRO, M. 1960: El Hombre ante la Historia. Madrid. Rialp.

AMORES, F. (1986): "Consideraciones metodológicas sobre las excavaciones en Itálica." Arqueología Espacial num 10. Epoca Romana y Medieval. Teruel. Colegio Universitario 
ANNEQUIN, J. (1982): “M.I. Finley et l'esclavage antique. Décrire et expliquer une forme d'explotation du travail" La Pensée num. 227.

1983: "Capital marchand et esclavage dans le procès de transformation des sociétés antiques" Colloque de Cortone. Pisa-Roma.

1985a: "Formes de contradiction et rationalité d'un système économique. Remarques sur l'esclavage dans l'antiquité" La Pensée, núm. 224.

1985b: "Comparatisme/comparaisons: Ressemblance et hétérogénéité des formes d'exploitation esclavagistes" Dialogues d"Histoire Antique núm 11.

1991: "Articulation entre production et circulation des biens dans l'Antiquité" La Pensée, núm. 280.

ARCE, J. (1976): "Sobre el supuesto Balbino de Itálica: una nota." Archivo español de Arqueología num 49. Madrid. CSIC.

1985: "Recensión a Calahorra. Madrid. Ministerio de Cultura.1984". Gerión. Madrid .

1989: "Estrabón sobre la Bética" En GONZÁLEZ, J.(ed): Estudios sobre Urso Colonia Iulia Genetiva. Sevilla. Alfar

ARCE, J.-PLÁCIDO, D. (1990): "Tendencias actuales y perspectivas de investigación en Historia Antigua." En VVAA: Tendencias en Historia. Encuentro en la Universidad Internacional Menéndez Pelayo. Pazo de Mariñán (Betanzos, La Coruña) Julio 1988. Madrid. CSIC.

BAENA, L. (1984): "Relieves romanos en la Provincia de Jaén." Archivo español de Arqueología num 57. Madrid. CSIC

BARANDIARÁN, I.-DELIBES, G.-FERNÁNDEZ-MIRANDA, M. 1990: "Situación actual y perspectivas en docencia e investigación para el área de Prehistoria." En VVAA: Tendencias en Historia. Encuentro en la Universidad Internacional Menéndez Pelayo. Pazo de Mariñán (Betanzos, La Coruña) Julio 1988. Madrid. CSIC

BARICH, B. (1982): "Archeologia teoretica. Il problema della teoria in Archeologia Prehistorica e nelle scienze umane." Origini num 11.

BATE, L.F. (1977): Arqueología y Materialismo Histórico. México. Ediciones de Cultura Popular. 1978: Sociedad, formacion economico-social y Cultura. México. Ediciones de Cultura Popular

BELTRÁN, J. 1991: "El mausoleo londinense del Procurator Iulius Classicianus." Habis num 22. Sevilla. Publicaciones de la Universidad

BENDALA, M.-PELLICER, M. (1977): "Nuevos hallazgos en el solar de la antigua Orippo (Dos Hermanas, Sevilla)." Habis num 8. Sevilla. Publicaciones Universidad

BERMEJO, J.C. (1983): "Etnografía castreña e historiografía clásica." Estudos de cultura castrexa e de Historia Antigua de Galicia. Santiago de Compostela

BIETTI, A. (1979): Metodi matematici e statistici applicati all'Archeologia e alla Paletnologia. Roma. Accademia Nazionale dei Lincei.

1982: Techniche matematiche nell'analisi dei dati archeologici. Roma. Accademia Nazionale dei Lincei

BINFORD, L.R-BINFORD, S.R.(eds) (1968): New perspectives in Archaeology. Chicago. Aldine

BINFORD, L.R. (1972): An archaeological perspective. London. 1983a: Working at Archaeology. New York. Academic Press. (1983b): In pursuit of the past. London. Thames and Hudson. 1989: Debating Archaeology. New York. Academic Press

BLANCO, A. (1984): La ciudad antigua (de la Prehistoria a los visigodos). Historia de Sevilla. Sevilla. Publicaciones Universidad. 
BORILLO, M.(ed) (1977): Raisonnement et methodes mathematiques en Archaeologie. Paris. Editions du CNRS

BRAVO, G. (1985): "Hechos y teoría en Historia (Antigua): Cuestiones teóricas en torno a un modelo-patrón de investigación." Gerión núm 3. Madrid

CAPOGROSSI, L.-GIARDINA, A.-SCHIAVONE, A. (eds) (1978): Analisi marxista e società antiche. Roma

CARANDINI, A. (1979): L'anatomia della scimmia. Torino. 1983: "Columella's vineyeard and the rationality of the Roman economy" Opus núm. 2.

(1985): Settefinestre, una villa schiavistica nell'Etruria romana. Modena.

1986: Schiavi in Italia. Gli strumenti pensanti dei Romani fra tarda Republica e medio Impero. Roma.

1989: "L'economia italica fra tarda republica e medio impero considerata dal punto di vista di una merce: il vino." Collection de l'Ecole Française de Rome núm. 114.

CARANDINI, A.-SETTIS, S. (1979): "Padroni et schiavi nell'Etruria romana." La villa de Settefinestre, dallo scavo alla mostra. Bari.

CARR, C.(ed) (1985): For concordance in Archaeology: Bridging data structure, quantitative techniques and theory. Westport

CERRILLO, E. (1988): "La aplicación de teorías del lugar central al territorio romano de Augusta Emerita." Arqueología Espacial tomo 12. Teruel. Colegio Universitario

CHOCLÁN, C.-CASTRO, M. (1988): "La Campiña del Alto Guadalquivir en los siglos I-II d.C. Asentamientos, estructura agraria y mercado." Arqueología Espacial tomo 12. Teruel. Colegio Universitario

CLARKE, D.L. (1968): Analytical Archaeology. London. Methuen

CLARKE, D.L.(ed) 1977: Spatial archaeology. London

CLAVEL-LEVEQUE, M. (1974a): "Les Gaules et les Gaulois: pour une analyse du functionnement de la Géographie de Strabon" Dialogues d'Histoire Antique núm. 1.

1974b: "Structures urbaines et groupes hétérogènes" Atti V Cesdir.

1974c: "Problèmes théoriques de l'histoire et sociétés antiques." Aujourd'hui l'histoire. Paris.

1975: "Pour une problématique des conditions économiques de l'implantation romaine dans le Midi gaulois." Cahiers ligures de préhistoire et d'archéologie núm. 24.

1976: "Urbanisation et cités dans l'Occident antique: voie privilégiée, imperialismes et transitions." Cahiers d'Histoire de l'Institut Maurice Thorez núm. 19.

1977a: "Imperialisme, Developpement et Transition: pluralité des voies et universalisme dans le modèle imperial romain.” La Pensée núm. 196.

(1977b): Espace des jeux dans le monde romain: hégémonie symbolique et pratique sociale. París.

CLAVEL-LEVEQUE, M.-FAVORY, F. 1977: "Pratique scientifique et théorie des sociétés de l'Antiquité". La Pensée num 192

CLEZIOU, S.-DEMOULE, J.P.-SCHNAPP, A. (1973): "Renouveau des méthodes et théorie de l'archéologie". Annales Economies, Sociétés, Civilisations num 1.

COURBÍN, P. (1988): What is Archaeology?An essay on the nature of Archaeological research. Chicago. University Press

CRIADO, F. (1988): “¿Que es un arqueólogo? ¿Que es la Arqueología?” Revista de Arqueología año IX num 82.

DÍAZ, M. (1993): "Theory and ideology in archaeology: Spanish archaeology under the Franco régime". Antiquity num 67 
DORAN, J.E.-HODSON, F.R.(eds) (1975): Mathematics and computers in Archaeology. Edinburgh DYSON, S.L. (1981): "A classical Archaeologist's response to the New Archaeology"BASOR num 242 Winona Lake.

1989: "Complacency and crisis in late twentieth-century Classical Archaeology." En CULHAM, P. LOWELL, E.(eds): Classics: a discipline and profession in crisis. New York.

1993: "From New to New Age Archaeology: Archaeological Theory and Classical Archaeology a 1990s perspective." American Journal of Archaeology num 97.

EISENSTADT, S.N. (1966): Los sistemas políticos de los Imperios. Madrid.

ESPINOSA, U. (1984): "El reinado de Cómmodo: subjetividad y objetividad en la antigua historiografía" Gerión, núm 2.

FAVORY, F. (1981): "Validité des concepts marxistes pour une théorie des sociétés de l'Antiquité". Klio num 63.

FERNÁNDEZ, V.-RUIZ ZAPATERO, G. (1984): "El análisis de territorios arqueológicos: una introducción crítica." Arqueología espacial num 8. Teruel. Colegio Universitario.

FERNÁNDEZ, V. (1985): "Las técnicas de muestreo en la prospección arqueológica." Ricus Vol 9. Num 3. Madrid.

FERREIRO, M. (1985): “Aproximación al establecimiento de un modelo matemático en un yacimiento arqueológico." Gades num 13. Cádiz. Diputación Provincial.

FINLEY, M.I. (1975): “Anthropology and the Classics”. En FINLEY, M.I.(ed): The use and Abuse of History. London.

1985a: The Ancient Historian and his Sources" En FINLEY, M.I.: Ancient History: evidence and models. London.

1985b: "Documents" en FINLEY, M.I.: Ancient History: evidence and models. London.

FREEDMAN, M.-DE LAET, S.J.-BARRACLOUGH, G. (1981): Corrientes de la investigación en las ciencias sociales. Madrid. Tecnos.

FONTANA, J. (1982): Historia. Análisis del pasado y proyecto social. Barcelona. Crítica.

GARCÍA, L. (1979): "La Península Ibérica y las tradiciones griegas de tipo mítico." Archivo español de Arqueología num 52. Madrid.ç

GIBBON, G. (1986): Explanation in archaeology. Oxford. Blackwell .

GÓMEZ, J. (1988): "Recensión a Crawforfd, M. (ed): Fuentes para la Historia de la Historia Antigua. Madrid. Taurus. 1986" Gerión num 6. Madrid.

GONZÁLEZ, C. (1986a): "Tartessos y las tradiciones literarias." Rivista de Studi Fenici num 14. Roma.

1986b: "Notas en torno a la aculturación en Tartessos." Gerión num 4. Madrid.

GRANT, E.(ed) (1986): Central Places, Archaeology and History. Sheffield. University Press.

HANNESTEAD, N. (1986): Roman art and imperial policy. Aarhus. Jutlan Archaeological Society Publications. num XIX.

HERNANDO, A. (1992): "Enfoques teóricos en Arqueología." Spal num 1. Sevilla. Publicaciones de la Universidad.

HIETALA, H.(ed) (1984): Intrasite spatial analysis in Archaeology. Cambridge. University Press.

HINDESS, B.-HIRST, P.Q. (1979): Los modos de producción precapitalistas. Barcelona. Península.

HODDER, I.-ORTON, C. (1976): Spatial analysis in Archaeology. Cambridge. University Press.

1978a: The spatial organisation of culture. London Duckworth.

1978b: Simulation studies in Archaeology. Cambridge. University Press.

1982: Symbolic and structural archaeology. Cambridge. University Press. 
1986: Reading the past. Current aproaches to interpretation in Archaeology. Cambridge. University Press.

HUMPHREYS, S. (1989): "Etudes classiques et anthropologie: Anthropologie et etudes anciennes". Actualité de l'Antiquité. París.

KATUZIAN, H. (1985): Ideología y método en Economía. Madrid. Blume.

KEAY, S. (1992): "The "romanisation" of Turdetania." Oxford Journal of Archaeology vol 11 num 3. Oxford.

KELLEY, J.H. (1988): Archaeology and the methodology of science. Albuquerque. New Mexico. University Press.

KELLER, I.-CLAVEL-LEVEQUE, M.-MILLOTE, J.P. (1976): "Discussion sur les formations economïques et sociales dans l'antiquité". Intervenciones. Actes du colloque sur l'esclavage (Besançon, 1973). Beçancon.

LUZÓN, J.M.-LEÓN, P. (1971): "Esculturas romanas de Andalucía." Habis num 2. Sevilla. Publicaciones de la Universidad.

MARTÍNEZ, M. (1989): Una revisión crítica de la Prehistoria española: la Edad del Bronce como paradigma. Madrid. Siglo XXI.

MCGUIRE, H.R. (1992): A marxist Archaeology. New York. Academic Press.

MONTANE, J. (1980): Marxismo y Arqueología. México. Ediciones de Cultura Popular.

MORA, R.-CARBONELL, E. (1990): "Estadística en Arqueología: el programa GTS." Archivo español de Arqueología num 63. Madrid. CSIC

MUELLER, J.W.(ed) (1975): Sampling in Archaeology. Tucson. University of Arizona Press.

NICOLET, C. (1988): "Economie des anciens, économie des modernes: remarques historiographiques." Rendre à César. Economie et sociétés dans la Rome antique. Editions Gallimard. Mesnilsur-l'Estrée.

ORTÓN, C. (1980): Mathematics in Archaeology. London. Collins.

PARAIN, Ch. (1965): "Rapports de production et développement des forces productives: l'exemple du moulin à eau." La Pensée núm 119.

1977: "Le développement des forces productives dans l'ouest du Bas-Empire." La Pensée num 196.

PASAMAR, G. (1991): "Corrientes, influencias y problemática en la historiografía contemporánea española." Studium num 3. Zaragoza. Colegio Universitario de Teruel.

PASTOR, M. (1983): "Aspectos sociales y económicos del Municipium Florentinum Iliberritanum." Archivo español de Arqueología. num 56. Madrid. CSIC.

PLA, A.J. (1982): La Historia y su método. Barcelona. Fontamara.

PONSICH, M. (1991): "Metodología para la lectura de un paisaje en la Antigüedad." Almoraima. Revista de Estudios Campogibraltareños. num 5. Algeciras.

PRESEDO, F. et alii (1986): Historia Antigua de España I. Protohistoria. Madrid. Cátedra.

PUERTAS, R.-RODRÍGUEZ, P. (1979): "La ciudad de Lacipo y sus monedas." Mainake num 1. Málaga.

RAMÍREZ, J.L. (1985): "Limitaciones inherentes a las fuentes literarias: Consecuencias de la guerra sertoriana para Calagurris." Gerión núm. 3. Madrid.

REMESAL, J. (1991): "La presencia de la Historiografía sobre Historia Antigua española en algunas revistas extranjeras." En ARCE, J.; OLMOS, R.(eds): Historiografia de la Arqueología y de la Historia Antigua en España (siglos XVIII-XX). Madrid. Ministerio de Cultura.

RODRÍGUEZ, P. (1978): “Municipium Barbesulanum.” Baetica num 1. Málaga.

RUIZ, A.-MOLINOS, M. (1984): "Elementos para un estudio del patrón de asentamiento en las 
campiñas del Alto Guadalquivir durante el Horizonte Pleno Ibero." Arqueología Espacial num 4. Teruel. Colegio Universitario.

RUIZ, A.-MOLINOS, M.-HORNOS, F. (1986): Arqueología en Jaén. Reflexiones desde un proyecto arqueológico no inocente. Jaén. Diputación Provincial.

RUIZ ZAPATERO, G. (1988a): "La prospección arqueológica en España: pasado, presente y futuro." Arqueología Espacial num 12. Teruel. Colegio Universitario.

(1988b): "Notas metodológicas sobre prospección en Arqueología." Revista de Investigación. Soria. Colegio Universitario.

1991: "Arqueología y Universidad. La reproducción del sistema" Revista de Arqueología. num 118 Madrid.

RUIZ ZAPATERO, G.-BURILLO, F. (1988): “Metodología para investigación en Arqueología territorial." Munibe num 6. San Sebastián.

RUIZ ZAPATERO, G.-CHAPA, T. (1990): "La Arqueología de la muerte: perspectivas teóricometodológicas." Actas del II Simposio sobre los Celtiberos. Zaragoza. Diputación Provincial.

SALMÓN, M.H. (1982): Philosophy and Archaeology. New York. Academic Press.

SAMSON, R. (1989):“Rural slavery, inscriptions, archaeology and Marx”. A response to Ramsay MacMullen's. "Late Roman slavery" Historia, vol. $38 \mathrm{n}^{\circ} 1$ Stuttgart.

SCOTT, E.(ed) (1993): Theoretical Roman Archaeology: first conference proceedings. Worlwide Archaeology Series num 4. Avebury. Ashgate publishing.

SHANKS, M.-TILLEY, C. (1987a): Reconstructing Archaeology: Theory and practice. Cambridge. 1987b: Social theory and Archaeology. Cambridge. Polity Press.

SHENNAN, S.J. (1988): Quantifying Archaeology. Edinburgh. University Press.

SNODGRASS, A.M. (1985): "The New Archaeology and the Classical Archaeologist" American Journal of Archaeology num 89.

SPRIGGS, M.(ed) (1984): Marxist perspectives in Archaeology. Cambridge.

STARR, C. (1965): "The credibility of Early Espartan History" Historia num 14.

VÁZQUEZ, J.M.-RISCH, R. 1991: "Theory in Spanish Archaeology" En HODDER, I. (ed): Archaeological Theory in Europe: the last three decades. London.

VICENT, J.M. (1982): "Las tendencias metodológicas en Prehistoria." Trabajos de Prehistoria num 39. Madrid. CSIC

VVAA 1972: Actes du Colloque 1971 sur l'esclavage. Centre de recherches d'Histoire Ancienne. Vol. 6. Annales littéraries de l'Université de Besançon, 140. Paris.

1974: Actes du Colloque 1972 sur l'esclavage. Centre de recherches d'Histoire Ancienne. Vol. 11. Annales littéraries de l'Université de Besançon, 163. Paris.

1976: Actes du Colloque 1973 sur l'esclavage. Centre de recherches d'Histoire Ancienne. Vol. 18. Annales littéraries de l'Université de Besançon, 182. Paris. 